\title{
LA RETÓRICA ESPECULATIVA - LA INTERPRETACIÓN DE JUDITH BUTLER de la Proposición Especulativa de Hegel
}

\author{
[THE SPECULATIVE RETHORIC - JUDITH BUTLER'S INTERPRETATION OF \\ HEGEL'S NOTION OF SPECULATIVE PROPOSITION]
}

\author{
Pamela Celeste Abellón * \\ Universidad de Buenos Aires, Argentina
}

\begin{abstract}
RESUMEN: El presente trabajo analiza la interpretación de la noción hegeliana de "proposición especulativa" que Judith Butler presenta en Sujetos del deseo. Reflexiones hegelianas en la Francia del siglo XX. Sostenemos que la filósofa norteamericana sostiene una interpretación retórica de la proposición especulativa porque comprende en términos retóricos el carácter especulativo de la proposición filosófica. Para ello, en primer lugar, presentamos la concepción hegeliana de la proposición especulativa, tal como ésta se desarrolla en el Prólogo a la Fenomenología del espíritu (1807). En segundo lugar, abordamos la interpretación de Butler. Por una parte, desarrollamos el carácter retórico de la proposición filosófica. Por otra parte, nos ocupamos de la idea de la apertura lógicosemántica de las determinaciones conceptuales que se deriva de la interpretación butleriana de la proposición en cuestión. Finalmente, en la conclusión, señalamos algunas limitaciones de la propuesta hermenéutica de Butler.
\end{abstract}

Palabras ClaVe: Proposición especulativa; Fenomenología del Espíritu; Judith Butler; Retórica

\begin{abstract}
This paper analyzes the interpretation of Hegel's notion of "speculative proposition" that Judith Butler presents in Subjects of Desire. Hegelian Reflections in Twentieth-Century France. We hold that the American philosopher maintains a rhetorical interpretation of the speculative proposition because she understands the speculative character of the philosophical proposition in rhetorical terms. In order to achieve this goal, first, we present the Hegelian conception of the speculative proposition, as it develops in the Prologue to the Phenomenology of the Spirit (1807). Second, we study Butler's interpretation. On the one hand, we develop the rhetorical character of the philosophical proposition. On the other hand, we deal with the idea of the logical-semantic opening of the conceptual determinations that derives from the Butlerian interpretation of the philosophical proposition. Finally, in the conclusion, we point out some limitations of Butler's hermeneutical proposal.

KEYWORDS: Speculative Proposition; Phenomenology of Spirit; Judith Butler; Rhetoric
\end{abstract}

\section{INTRODUCCIÓN}

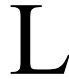

a proposición especulativa (spekulativer Satz) es un concepto que Hegel tematiza de manera explícita en la "Vorrede" a la Phänomenologie des Geistes (1807). ${ }^{1}$ En términos generales, aquella es la proposición filosófica por excelencia, puesto que expresa (drückt aus) y expone (stellt dar) la verdad en su carácter especulativo (Ph., 20-21, 43-46; Hoffmann [2004] 2014, pp. 206-211). ${ }^{2}$

* Doctora (PhD) en Filosofía por la Universidad de Buenos Aires, Argentina. Docente en la Facultad de Filosofia y Letras de la Universidad de Buenos Aires y Becaria posdoctoral del Consejo nacional de investigaciones cientificas y técnicas (CONICET).m@ilto: abellonpamela@gmail.com 
Hay dos cuestiones generales que, en principio, dificultan sopesar con exactitud la función y el valor que la proposición especulativa tiene en la filosofía hegeliana. La primera consiste en que lo que nos llega como la "Vorrede" a la $P h$. es un texto publicado originalmente como Prólogo al System der Wissenschaft, del que la "Phänomenologie des Geistes" constituía sólo su primera parte. ${ }^{3}$ La segunda es que, en su etapa de madurez, Hegel dejó de comprender a la Fenomenología del espíritu como la primera parte del Sistema. En 1831, cuando corrige la $P h$. para su segunda edición, elimina las referencias que la ubican como la "primera parte del sistema" $(P h ., 24-25)$. Además, el incompleto Sistema de la ciencia de juventud fue "reemplazado" por la Enzyklopädie der philosophischen Wissenschaften im Grundrisse (EPW). En ésta, la "Phänomenologie des Geistes" forma parte de "Die Philosophie des Geistes", tercera y última parte del Sistema, ubicándose específicamente en el segundo momento del Espíritu subjetivo (der subjektive Geist), entre la "Anthropologie" y la "Psychologie" $(E P W, \S \S 413-439)$.

En comparación con otras nociones y temáticas del amplio corpus hegeliano, la proposición especulativa ha recibido poca atención por parte de la Hegelsforschung. Según una línea hermenéutica, la proposición especulativa tiene un papel central en el Sistema hegeliano en general, el cual puede interpretarse a la luz de aquella (Lau 2006; Nuzzo 2006; Simon [1966] 1982, pp. 225-228, 241-268; Surber 1975; Wohlfart 1981). Otro lineamiento interpretativo entiende que Hegel abandona la "doctrina" de la proposición especulativa entre 1810-1811, cuando descubre el valor especulativo del silogismo y comienza a considerar que, debido a su forma, toda proposición es incapaz de expresar la verdad filosófica (Duque 2011, p. 15; 1990; Düsing 1986, pp. 15-38; McCumber 1993, pp. 135-136). ${ }^{4}$

El presente trabajo no se dedicará a tomar parte en este debate, tarea que requeriría un tipo de abordaje del problema que excede el tratamiento de la proposición especulativa que aquí llevaremos a cabo. Por el momento, nos centraremos en el concepto de la proposición especulativa tal como Hegel lo presenta en la "Vorrede" a la $P h$. y en función del rol que ocupa en la exposición científica de la experiencia de la conciencia. Dentro de este marco, nos proponemos abordar la novedosa y original interpretación que, al respecto, Judith Butler elabora en Subjects of Desire. Hegelian Reflections in Twentieth-Century France $(S D) .^{5}$

La primera cuestión sobre la que Butler llama la atención en el Capítulo I de $S D$, "Desire, Rhetoric, and Recognition in Hegel's Phenomenology of Spirit", se refiere al propio lenguaje de Hegel, problemática que retoma en el prólogo de 1998 destinado a la segunda edición el libro ( $S D$, pp. 13-15, 49-52). Según la filósofa, el análisis de la $P h$. requiere primero detenerse en el modo idiosincrásico en que Hegel desarrolla los contenidos filosóficos. Cualquier lector de la $P h$. advierte la dificultad que esta obra conlleva no sólo en lo que respecta a su contenido, sino también a su estructura narrativa. Al respecto, Butler sostiene:

El análisis [de la] Fenomenología del espíritu requiere, como paso preliminar, examinar una cuestión más amplia: cómo se presentan y "argumentan" los temas filosóficos en este, por momentos, intrincado texto. Escribo la palabra "argumentan" entre comillas no para desestimar la clase de argumentación desplegada por Hegel, sino para llamar la atención sobre la idiosincrasia de su forma. Después de todo, la Fenomenología del espiritu es una Bildungsroman, un optimista relato de aventura y esclarecimiento, una peregrinación del espíritu, y a primera vista no resulta claro el modo en que la estructura narrativa adoptada por Hegel expone su argumentación metafísica. Es más, la estructura oracional utilizada por Hegel parece desafiar las leyes de la gramática y poner a prueba la imaginación ontológica más allá de los límites a los que está habituada. ( $S D, \mathrm{p}$. 
49).

Muy pocos estudios se ocuparon de las consideraciones que Butler realiza sobre el lenguaje hegeliano y aquellos que sí lo hicieron comprendieron de manera insuficiente las ideas que ella expone sobre este tema. En líneas generales, estas investigaciones entienden que la autora hace referencia a una cuestión esencialmente estilística (Burgos 2008, pp. 14-16; Salih 2012, pp. 13-15; Vicki 2006, pp. 5-6). En estos términos, por ejemplo, Salih sostiene que "Butler describe el estilo de la prosa (prose style) de Hegel" (2012, p. 13). Una excepción a la interpretación "estilística" es la que presentan Casale y Femenías:

$[\mathrm{P}]$ ara Butler, el escenario donde se despliegan estas operaciones [sc. la superación de las oposiciones] no es más que un universo lingüístico de modo tal que esas operaciones van a estar atravesadas por el discurso, y por ello el movimiento puesto en juego en esas operaciones se puede concebir como retórico (2009: 26).

Estos autores, sin embargo, no especifican en qué consistiría dicho "universo discursivo". En ambos casos, estas interpretaciones consideran que Butler hace alusión a una cuestión esencialmente estilística o discursiva que tendría como objetivo mostrar la complejidad del movimiento de lo real partir de la exposición de la dificultad lingüística. La mayor deficiencia que encontramos en este tipo de lecturas es que no reparan en que el análisis de Butler sobre el problema del lenguaje hegeliano tiene por objeto de estudio la proposición especulativa (speculative proposition) que Hegel presenta en la "Vorrede" a la $P h$.

En contraposición a dichos posicionamientos, el presente trabajo se propone mostrar que Butler sostiene una interpretación retórica de la proposición especulativa porque comprende en términos retóricos el carácter especulativo de la proposición filosófica. Para ello, en primer lugar, presentamos la concepción hegeliana de la proposición especulativa, tal como ésta se desarrolla en el "Vorrede" a la $P h$. En segundo lugar, abordamos la interpretación retórica de Butler sobre de la sentencia especulativa. Por una parte, desarrollamos el carácter retórico de la proposición filosófica. Por otra parte, analizamos idea de la apertura lógico-semántica de las determinaciones conceptuales que se deriva de su lectura sobre la proposición en cuestión. Finalmente, en la conclusión, señalamos algunas limitaciones de la propuesta hermenéutica de la filósofa norteamericana.

\section{LA PROPOSICIÓN ESPECULATIVA EN LA PH}

El contexto general en el que Hegel expone la noción de "proposición especulativa" (spekulativer Satz) en la "Vorrede" a la $P h$. es la "presentación" de la filosofía especulativa. ${ }^{6}$ En términos más específicos, tal exposición tiene lugar en medio del tratamiento hegeliano sobre la distinción entre el pensar concipiente (begreifendes Denken), propio de la Ciencia (Wissenschaft), y el pensar representador (vorstellendes Denken) o el raciocinar (das Rässonieren), reflexión externa del Entendimiento (Verstand) respecto del movimiento de las determinaciones conceptuales (Ph., 43-46).

El pensar representador es el que es incapaz de cumplir las exigencias del estudio de la Ciencia. Este último requiere que el pensar tome sobre sí el esfuerzo del Concepto (Begriff). En efecto, el que la sustancia sea sujeto significa, entre otras cosas, que "en ella [...] todo contenido es su propia reflexión dentro de sí (ist aller lnhalt seine eigene Reflexion in sich)" (Ph., 39). Por esto, el pensar filosófico debe fijarse en las determinaciones simples del concepto tal como éstas son, es decir, como 
automovimientos (Selbstbewegungen) (Ph., 41). Pero el raciocinar, cuyo yo sapiente es el yo vacío, el sí mismo formal del Entendimiento (Verstand), es libre respecto del contendido conceptual. En lugar de hundirse en él, se pone por encima del concepto y pretende mantener el contenido en determinaciones fijas y estables. Dado su criterio de verdad, que es la forma vacía del principio de identidad $(\mathrm{A}=\mathrm{A})$, el comportamiento raciocinante (räsonierendes Verhalten) es negativo respecto del contenido: resiste el movimiento del concepto refutando y aniquilando las determinaciones contradictorias que éste conlleva. Cuando, frente al movimiento conceptual, el pensar representador se obstina en hacer persistir la estabilidad del contenido en determinaciones fijas y afirma "que algo no es así"7, se muestra él mismo como una intelección vacía y formal de lo negativo, que no incorpora dentro de sí la negatividad de la que surge lo positivo de un nuevo contenido. En este sentido, al no poder aprehender el concepto y sus determinaciones en su movimiento, el raciocinar es por su propia naturaleza incapaz de emprender la tarea científica.

El modo proposicional mediante el cual el pensar representador explica o expresa (drückt aus) su contenido es la "proposición en general" (Satz überhaupt), que pone de relieve la aprehensión externa y libre respecto del concepto característica del modo de conocer de la conciencia natural. La proposición en general mantiene la distinción entre el sujeto y el predicado. El primero constituye la base en la que adhiere el contenido, sus accidentes, por medio del predicado. Se trata del lenguaje proposicional ( $\mathrm{S}$ es $\mathrm{P}$ ), en el que el sujeto, permaneciendo fijo, adquiere su determinación o contenido por medio de la predicación, siendo el yo sapiente, es decir, algo externo a los componentes de la proposición misma, quien enlaza el sujeto y el predicado en cuestión (Ph., 42-43). A propósito de esto, Hegel brinda el siguiente ejemplo:

La menesterosidad por representar lo absoluto como sujeto se servía de proposiciones como: Dios es lo eterno [...] En tales proposiciones, lo verdadero tan sólo está puesto directamente como sujeto, pero no está presentado como el movimiento del reflexionarse hacia dentro de sí mismo. En una proposición de este género se empieza con la palabra: Dios. Por sí misma, es un sonido sin sentido, un mero nombre, sólo el predicado dice lo que él es, lo llena dándole cumplimiento y significado; el comienzo vacío se hace saber efectivo solamente en ese final [...] El sujeto se acepta como punto fijo al que adhieren, como a su asidero, los predicados mediante un movimiento que pertenece a aquel que sabe acerca del sujeto, y que no es considerado como perteneciente al punto mismo; pero, por medio de ese movimiento, lo único que quedaría expuesto es el contenido como sujeto. Este movimiento, por el modo como está hecho, no puede formar parte de él; mas, una vez presupuesto ese punto, tampoco puede estar hecho de otro modo, sólo puede ser exterior. Por eso, aquella hipótesis anticipada de que lo absoluto es sujeto, no sólo no es la realidad efectiva de este concepto, sino que llega incluso a hacerla imposible, puesto que pone el concepto como punto en reposo; cuando la realidad efectiva es el automovimiento. (Ph., 21; el subrayado es del original)

El saber del raciocinar, según explica Montecinos (2012), se fundamenta en algo fijo y estable que se tiene por consabido (bekannt), y se constituye en base a la identidad entre el yo sapiente y el sujeto gramatical de la proposición, al que el autor denomina "sujeto-objetual". El sujeto raciocinante proyecta el sí mismo formal que él es como el sujeto gramatical fijo de la proposición, al que adhiere exteriormente el contenido por medio de los predicados (conceptos universales) según su propia representación de lo que la cosa es, una identidad formal $(\mathrm{A}=\mathrm{A})$, representación que tiene su expresión en proposiciones semejantes $(\mathrm{S}=\mathrm{S})$. Esta exterioridad de la 
predicación pone de relieve que para el raciocinar las determinaciones carecen de necesidad, puesto que el yo sapiente se posiciona por encima del sujeto-objetual y lo determina considerando solamente sus propios presupuestos formales y su criterio de identidad. Esto último explica que el raciocinar refute y aniquile las determinaciones o predicados contradictorios que dicen algo diferente de lo que es el sujeto idéntico a sí mismo (- A). Mediante este proceder, el comportamiento raciocinante reduce el sujetoobjetual a una forma vacía y vuelve a sí mismo como yo sapiente-formal. Por lo tanto, el raciocinar es una reflexión externa respecto del contendido del objeto, en la que el yo vacío no sale de sí mismo (2012, pp. 57-59).

El pensar que, en contraposición al raciocinar, sí toma sobre sí el esfuerzo del concepto es el pensar concipiente. Éste se sumerge en el contenido del concepto y en el movimiento propio de sus determinaciones, siendo inmanente a este último ( $P$ h., 4142). Este pensar, a diferencia del pensar representador, es la aprehensión positiva del movimiento conceptual, para la cual lo negativo forma parte del contenido mismo y de su propia determinación (Ph., 42-43). Es en el comportamiento de este pensar en el que radica la actividad (Tätigkeit) propia del saber filosófico, la cual:

$[\mathrm{N}]$ o es la reflexión dentro sí sacada a partir del contenido; la ciencia no es ese idealismo que sustituye al dogmatismo asertivo bajo la forma de un dogmatismo aseverador o de un dogmatismo de la certeza de sí mismo: sino que, viendo el saber que el contenido retorna a su propia interioridad, ocurre más bien que su actividad tanto se ha sumergido en el contenido - pues ella es el sí-mismo inmanente de éste- cuanto, a la vez, ha retornado dentro de sí - pues ella es la pura igualdad-a-sí-mismo en el ser otro-. (Ph., 39-40; el subrayado es del original)

El saber del contenido que es Concepto es, según Hegel señala en la "Vorrede", la necesidad lógica y lo especulativo, que constituye la propia naturaleza del método científico. Este último no es un método formal y separado del contenido, sino que es inmanente al propio movimiento del concepto, que halla su propia exposición (Darstellung) en la Filosofía especulativa (Ph., 40-41).

La explicación o expresión del contenido por parte del pensar concipiente tiene lugar mediante la proposición especulativa (spekulativer Satz), la cual destruye la forma proposicional característica del comportamiento raciocinante. Al respecto, Hegel sostiene:

\begin{abstract}
Al ser el concepto el sí-mismo propio del objeto, sí-mismo que se expone (darstellt) como el devenir de este objeto, no es un sujeto en reposo que, sin moverse, soporta los accidentes, sino que es el concepto que se mueve y que recoge dentro de sí sus determinaciones. En este movimiento, ese sujeto mismo en reposo se viene abajo; queda subsumido en las diferencias y el contenido, y constituye, más bien, la determinidad, es decir, el contenido diferenciado, así como el movimiento de éste, en lugar de permanecer erguido frente a él. El suelo firme que el raciocinar tiene en el sujeto en reposo, entonces, se tambalea [...] El contenido ya no es, entonces, predicado del sujeto, sino que es la substancia, es la esencia y el concepto de aquello de lo que se está hablando. ( $P h ., 42-43$; el subrayado es del original)
\end{abstract}

Con la proposición especulativa, el pensar representador sufre, dice Hegel, un contragolpe (Gegenstoß). El sujeto que dicho pensar pone como fundamento y soporte al que adhieren los predicados pasa a ser, por el movimiento dialéctico del concepto, él mismo predicado. De este modo se invierten las relaciones entre el sujeto y el predicado que el raciocinar pretende mantener fijamente distinguidas, viéndose él mismo limitado en esta tarea y en su comportarse. Hegel resume esta cuestión de la siguiente manera: 
Formalmente, lo que hemos dicho puede expresarse así: la naturaleza del juicio, o de la proposición en general, que encierra dentro de sí la diferencia de sujeto y predicado, es destruida por la proposición especulativa, y la proposición de identidad en la que se convierte la primera contiene el contragolpe a aquella relación [...] En la proposición filosófica [sc. la proposición especulativa], la identidad del sujeto y del predicado no debe anular la diferencia entre ellos, expresada por la forma de la proposición, sino que su unidad debe brotar como una armonía. (Ph., 43-44)

La Ciencia no debe mezclar el modo proposicional especulativo y el raciocinante. La exposición filosófica (philosophisches Exposition) se realiza en el primero de los modos proposicionales mencionados. Esto no quiere decir, sin embargo, que tal exposición anule todo tipo de vínculo con la proposición en general. El que ésta se revele como insuficiente respecto del saber y quede en suspenso es algo que debe ser proferido (ausgesprochen) y expuesto (dargestellt). La exposición especulativa (spekulative Darstellung) es precisamente el proferir el movimiento dialéctico de las determinaciones conceptuales y, por lo tanto, el contragolpe de la proposición en general $(P h ., 45) .{ }^{8}$ La exposición filosófica expone la destrucción de la proposición en general, es decir, la reflexión del contendido conceptual que el raciocinar rechaza e intenta fijar. Esta reflexión interna del contenido, el automovimiento de las determinaciones del concepto, es lo que la Wissenschaft der Logik (WL) expone en su forma pura, y la $P h$. según su aparecer para la conciencia (Montecinos 2012, p. 59).

Cubo Ugarte (2010) propone una manera de explicar esto último que puede resultar esclarecedora dada su simplicidad. Según el autor, en la $P h$. hay un doble registro de lenguaje. El primero es el lenguaje proposicional, la proposición en general, que es propio de la conciencia natural y del lector de la $P h$. no iniciado aún en la filosofía especulativa, el cual precisamente toma el posicionamiento de la conciencia natural. El segundo registro lingüístico es el lenguaje especulativo, la proposición filosófica. Este registro corresponde a la filosofía especulativa y pertenece a aquel que ha llegado al Saber absoluto y conoce la necesidad del movimiento lógico de las determinaciones del concepto y del tránsito fenomenológico, Hegel, en primer lugar, y el lector de la $P h$. que conoce el sistema hegeliano, en segundo lugar. Estos últimos, Hegel y el filósofo especulativo, constituyen el "nosotros fenomenológico" de la $P h$., que capta y ordena de manera sistemática las diferentes figuras de la conciencia según la necesidad científica que sucede a espaldas de la conciencia natural. Dicha necesidad se pone de manifiesto expresamente en los párrafos de la $\mathrm{Ph}$. denominados Wir-Stücke, que son aquellos en los que Hegel distingue de manera explícita aquello que es para la conciencia y aquello que es "para nosotros" (2010, pp. 35-41). A propósito de esto, Hegel afirma en la "Einleitung":

Esto se presenta aquí de tal manera que, en tanto que lo que primero aparecía como el objeto se degrada, a ojos de la conciencia, a un saber acerca de él, y en tanto que lo en sí se convierte en un ser de lo en si para la conciencia, este ser es el nuevo objeto, con el cual también entra en escena una nueva figura de la conciencia, para la cual la esencia es algo distinto de lo que lo era para la figura precedente. Es esta circunstancia la que guía toda la serie de figuras de la conciencia en su necesidad. Sólo esta necesidad misma, o la emergencia del nuevo objeto que se presenta a la conciencia sin que ésta sepa lo que le acontece, es lo que sucede para nosotros (für uns), por así decirlo, como a sus espaldas. Entra así en su movimiento un momento del ser en sí, o del ser para nosotros (Fürunsseins), que no se expone (darstellt) para la conciencia, la cual está prendida en la experiencia misma; pero el contenido de lo que emerge ante nosotros es para ella (für es), y nosotros sólo concebimos la parte formal del 
mismo, o su puro originarse; para ella, esto que se ha originado es sólo como objeto, para nosotros es, a la vez, como movimiento y devenir. (Ph., 61; el subrayado es del original)

La emergencia de lo que es para nosotros, la necesidad del movimiento conceptual, no se expresa -repitamos- de forma independiente al lenguaje proposicional propio de la conciencia natural. Como hemos señalado anteriormente, la exposición filosófica es la exposición (Darstellung) del movimiento del concepto en su necesidad, exposición que se desarrolla en cuanto la proposición especulativa profiere el contragolpe de la proposición en general. Sobre esta cuestión, Cugo Ugarte subraya:

Gracias al doble lenguaje que se despliega a partir del nosotros fenomenológico se puede distinguir entre lo meramente mentado o supuesto (Gemeintem) por la conciencia en cada una de sus experiencias y lo realmente dicho (Gesagtem) o acontecido en ellas [...] Que el sentido de cada una de estas experiencias sea algo provisional, o sea algo que no puede ser completamente dicho no es algo meramente accidental a una determinada figura de la conciencia, sino que es algo esencial a la forma misma de la proposición [...] No puede ser dicho debido a la forma misma de la proposición. Pues bien, es esta forma de la proposición lo que intenta violentar Hegel a través de lo que él denomina la proposición especulativa. La proposición especulativa no pretende decir algo de algo, sino más bien mostrar de soslayo a través de la forma misma de la proposición los límites inmanentes a todo decir proposicional [...] El uso especulativo del lenguaje no puede renunciar al lenguaje proposicional, porque lo necesita para transcurrir a su través. (2010, pp. 41-42; el subrayado es del original) ${ }^{9}$.

La peculiaridad del proceder de la filosofía especulativa explica la dificultad que los lectores no iniciados en la Ciencia y atados al comportamiento raciocinante hallan en los escritos de filosofía. Pretenden encontrar en la proposición filosófica la relación habitual entre el sujeto y el predicado característica de la proposición en general que se corresponde con su forma del saber. Pero en tanto que su comportamiento y su opinión se ven destruidos por el propio contenido filosófico, el concepto y el movimiento de sus determinaciones, dichos espíritus se ven obligados, tras la corrección del concepto, a volver sobre las proposiciones y captarlas en su modo especulativo ( $P h ., 44-46)$.

\section{LA INTERPRETACión DE JUdith BUTLER}

\subsection{El carácter retórico de la proposición especulativa}

El análisis que Butler realiza sobre el lenguaje de la $P h$. toma en consideración la inseparabilidad que Hegel subraya en la "Vorrede" entre la exposición conceptual de la filosofía especulativa y el modo proposicional científico. Al respecto, sostiene: "La verdad (truth) no es lo mismo que el relato (narrative) ofrecido en la Fenomenología; sin embargo, sólo se manifiesta mediante su exposición (it is made manifiest only through its exposition)" (SD, p. 13; traducción modificada). ${ }^{10}$ Según Butler, la dificultad que conlleva la comprensión de tal exposición para muchos lectores de la $P h$. se debe a que abordan esta obra con el supuesto de que el texto describe una realidad estable mediante proposiciones regidas por la lógica proposicional clásica y cuyas partes refieren a entendidas ontológicas que se corresponden con ellas. La noción hegeliana de "proposición en general" como modo proposicional del pensar representador o comportamiento raciocinante es comprendida por Butler en términos de lenguaje 
descriptivo y referencial, propio de la perspectiva del Entendimiento (Understanding / Verstand). Desde el posicionamiento de este último, que fija el sujeto gramatical en un significante unívoco, el lector de la $\mathrm{Ph}$. pretende encontrar en las oraciones hegelianas la relación habitual entre el sujeto y el predicado, según la cual el primero soporta los accidentes que el segundo introduce. Sin embargo, a medida que avanza la lectura, el lector repara en que los sujetos gramaticales son intercambiables con sus predicados, o que ellos se articulan con verbos que luego son negados e invertidos en proposiciones subordinadas. De este modo, el propio contenido del texto y su movimiento enfrenta al lector con la perplejidad y obstinación del lenguaje descriptivo (descriptive lenguage), y lo obliga a poner en duda y reexaminar bajo una nueva luz los presupuestos y las convicciones adquiridas hasta el momento ( $S D$, pp. 14-15, 49-50). En este sentido, Butler afirma: "Si ponemos en cuestión los supuestos del Entendimiento a los que nos invita la prosa, experimentamos el incesante movimiento de la oración que constituye su propio significado" (SD, p. 51).

En contraposición a las proposiciones descriptivas, las especulativas "ponen en acto (enact) los significados (meanings) que portan (convey); de hecho, muestran (show) que aquello que 'es' (what 'is') sólo es en la medida (only is to the extent) en que es puesto en acto (enacted)" (SD, p. 50; el subrayado es del original). ${ }^{11}$ Mediante esta formulación contemporánea, lo que Butler intenta poner de relieve es que la proposición especulativa (speculative proposition) no sigue la lógica referencial del lenguaje descriptivo. En este mismo sentido afirma: "Como un verso de una poesía que nos hace detener y nos obliga a pensar que la manera (way) en que está dicho es esencial para lo que (what) dice, las oraciones hegelianas llaman la atención sobre sí mismas retóricamente (rhetorically)" (SD, p. 50; el último subrayado es nuestro).

Butler entiende, en efecto, el carácter especulativo de la proposición filosófica en términos retóricos. Esta última desafía las leyes gramaticales de la lógica proposicional. En oposición a la fijeza de la proposición descriptiva, el movimiento de la oración especulativa hace que lo conocido hasta el momento por el lector se torne extraño $(S D$, p. 13). Esto se debe, como hemos visto anteriormente, al hecho de que la proposición filosófica expresa el movimiento dialéctico del concepto y sus determinaciones, poniendo en duda la comprensión habitual de las relaciones lógicas -fundamentalmente de aquellas que mantienen la distinción clásica del sujeto y el predicado- y desafiando la concepción metafísica que éstas conllevan ( $S D$, pp. 13-14, 49-50).

La retoricidad de la proposición filosófica o, como la denominamos aquí, la "retórica especulativa", se pone especialmente de manifiesto por el lugar gramatical de la "negación" (negation), término con el que Butler se refiere no sólo a las partículas gramaticales negativas sino también y fundamentalmente a la negatividad propia del desarrollo del concepto. ${ }^{12}$ Aunque la negación, argumenta Butler, adquiere diversas formas y sentidos a lo largo de la $P h$., aquello que la caracteriza es que cumple un papel esencial en el despliegue de la verdad filosófica $(S D$, p. 14). Recordemos que para Hegel, según hemos visto anteriormente, el pensar concipente es la intelección positiva del movimiento del concepto, para la cual lo negativo se concibe como parte del contenido conceptual mismo y de su propia determinación. Al respecto, Butler sostiene:

Estas funciones de la "negación" dan lugar a las bromas que suelen hacer respecto de Hegel los analistas contemporáneos, quienes insisten en que hay que volverlo claro y sencillo o rechazarlo de pleno. Sin embargo, Hegel tiene otra idea en mente cuando afirma, por ejemplo, en la Fenomenología, que la proposición especulativa (speculative proposition) destruye la naturaleza general de la proposición [...] Advertimos que el lenguaje que, según creíamos, hablaba de la 
realidad de la negación participa de la actividad misma, tiene su propia función negadora y, de hecho, está sujeto también a la negación. Así, el lenguaje del texto exhibe su propia retoricidad (rhetoricity), y descubrimos que la cuestión de la lógica y la retórica no pueden ser en modo alguno disociadas (the question of logic and that of rhetoric are indissociable from each other). De manera similar, no es posible hablar de cognición al margen de la práctica de la lectura: no es posible separar la temporalidad del concepto de la temporalidad de la lectura. ( $S D$, pp. 14-15; el subrayado es nuestro)

El movimiento especulativo de la proposición, al que Butler tematiza en términos de "inversión retórica" (rhetorical inversion), muestra, según ella, la naturaleza esquiva (elusive nature) tanto del sujeto gramatical como del sujeto humano, es decir, de la conciencia que adquiere diversas configuraciones a lo largo de su desarrollo fenomenológico hasta alcanzar el Saber absoluto. Cabe volver aquí sobre una idea central que Hegel afirma en la "Einleitung" y que consiste en que la $P h$., como ciencia de la experiencia de la conciencia, emprende la exposición del saber que aparece (die Darstellung des erscheinenden Wissen), es decir, la exposición del camino de la duda y la desesperación que recorre la conciencia natural hasta el Saber absoluto (Ph., 55-56). El método de ejecución (die Methode der Ausführung) es el que rige esta exposición, representada, dice Hegel, "como comportamiento de la ciencia respecto del saber que aparece, y como investigación y examen de la realidad del conocer" (Ph., 58; el subrayado es del original). El método en cuestión no consiste en la aplicación de una pauta exterior puesta por un sujeto sapiente externo al desarrollo de las determinaciones conceptuales implicadas en la experiencia de la conciencia, sino que el método es inmanente al movimiento dialéctico de la conciencia y del saber o, en otros términos, es su misma exposición (Darstellug) mediante la proposición especulativa. En base a esta idea, Butler entiende que la proposición filosófica refleja y representa el movimiento progresivo de la experiencia de la conciencia, es decir, las inversiones que ésta realiza en su camino hacia el Saber absoluto ( $S D$, p. 51). A propósito de esto, sostiene:

Contra la compulsión del Entendimiento a fijar el sujeto gramatical en un significante unívoco y estático, las oraciones hegelianas indican que sólo es posible comprender al sujeto en su movimiento [...] El sujeto gramatical nunca es idéntico a así mismo, sino que siempre y solamente es él mismo en su movimiento reflexivo. ( $S D$, p. 50$)$

Las inversiones retóricas, en el sentido recién mencionado, muestran el desplazamiento (displacement) del sujeto gramatical y del sujeto humano debido a que el movimiento proposicional especulativo, que refleja el desarrollo dialéctico de la experiencia de la conciencia, pone de relieve que no es posible considerar a aquellos en términos simples y estables. Cuando el lector, explica Butler, obtiene una noción precisa del sujeto mediante indicaciones gramaticales y semánticas determinadas, observa prontamente que el sujeto se desplaza y se convierte en algo otro, tornándose extraño a la luz del nuevo movimiento gramatical y fenomenológico ( $S D$, p. 52). En este sentido, en el "Preface to the Paperback Edition", Butler afirma que lo que ofrece la $\mathrm{Ph}$. es "una definición [sc. del sujeto] en desplazamiento" (SD, p. 21).

Butler refuerza esta idea mediante algunas consideraciones sobre la proposición que afirma una de las tesis fundamentales de Hegel: "la sustancia es sujeto". La idea de que la sustancia es sujeto sólo se explica mediante la exposición (Darstellung) del sistema de la Ciencia. Por lo tanto, la oración que la enuncia debe ser leída a la luz del propio desarrollo del concepto y no al margen de éste. Butler considera que la proposición en cuestión no sólo sugiere lo que son la sustancia y el sujeto, sino que 
remite fundamentalmente a la totalidad del movimiento de las determinaciones del concepto. La cópula "es" de dicha oración debe comprenderse en términos de "deviene", donde "devenir" significa un movimiento dialéctico, progresivo y cíclico en el que cada uno de los términos, la "sustancia" y el "sujeto", llega a ser él mismo en tanto que es también el otro ${ }^{13}$. En este sentido, el "es" se expresa como asiento del movimiento conceptual y de la plurivocidad semántica: "Leer la oración [sc. 'la sustancia es sujeto'] correctamente implicaría leerla en forma cíclica o hacer entrar en juego la variedad de significados parciales ínsitos en cualquier lectura dada" (SD, p. $50)$.

La última cuestión a la que hace referencia el análisis de Butler sobre el lenguaje de la $\mathrm{Ph}$. es la concepción holística del significado. Las oraciones hegelianas, al tiempo que ponen en acto los significados que expresan o portan, indican (indicate) de manera retórica lo que no es dicho en la proposición pero que es esencial para que ésta adquiera significado. Cada una de ellas remite al contexto semántico más amplio en el que tiene que ser interpretada, a una multiplicidad de significados que se determinan entre sí de manera continua. Esta multiplicidad y determinación recíproca no es estática ni unilateral, sino el movimiento mismo del desarrollo del concepto (SD, p. 52). Esto quiere decir que aquel contexto en el que cada proposición y los términos que la componen adquieren su verdadero sentido es la totalidad del desarrollo de las determinaciones conceptuales ${ }^{14}$. De esta manera, según la interpretación de Butler, la retórica especulativa se presenta como la exposición misma del concepto en el automovimiento y las inversiones retóricas de sus propias determinaciones.

\subsection{La apertura lógico-semántica}

La interpretación que Butler realiza de la proposición especulativa incluye un problema que ella no desarrolla por sí mismo, pero respecto del cual sí da indicaciones sobre una posible respuesta. El tema en cuestión es el de cómo comprender la relación entre la totalidad lógica y semántica de las determinaciones conceptuales a la luz de la proposición especulativa. A propósito de esta cuestión, Butler sostiene:

"Sustancia es sujeto" sugiere no sólo qué es la sustancia, qué es el sujeto y cuál es el significado de la cópula, sino también que no hay razonamiento (ratiocination) de posibles significados que pueda capturar todos los significados que sugiere la oración. Los tres términos significan de la manera indefinida (signify indefinitely), puesto que cada uno de ellos exige una continua especificación y revisión. Conocer el significado de esa oración es conocer el significado del sistema postulado por Hegel, y ningún sujeto vivo puede conocer ese significado de una vez y para siempre (that meaning cannot be known once and for all by any living subject) [...] Tal multiplicidad de significados no es estática, según Hegel, sino que es la esencia del devenir, del movimiento mismo (the essence of becoming, of movement itself). (SD, pp. 51-52; traducción modificada) ${ }^{15}$.

El hecho de que el significado completo del sistema hegeliano no pueda ser conocido y que, por lo tanto, tampoco pueda alcanzarse el sentido definitivo de "la sustancia es sujeto" no radica ni en una deficiencia del sistema científico ni en una incapacidad del filósofo especulativo. La razón de tal imposibilidad consiste en la apertura del sistema de Hegel. Esta apertura, defendida por Butler, tiene como base su propia interpretación temporal de lo Absoluto ${ }^{16}$. Al respecto, sostiene:

Aunque a veces se categoriza a Hegel como el filósofo de la totalidad, de la completitud sistemática y la autonomía autosuficiente, no está claro que la 
totalidad metafísica que postula constituya un sistema finito. De hecho, la paradoja irreductible de la metafísica hegeliana parece radicar en la apertura (openness) de este sistema, aparentemente exhaustivo (all-inclusive). Que una metafísica sea a la vez completa e infinita significa que esa infinitud ha de ser incluida en el sistema mismo, pero la "inclusión" como relación espacial es una manera deficiente de describir la relación del infinito con el sistema mismo. Poder pensar el absoluto de Hegel -el infinito y lo sistemático a un tiempo- implica pensar más allá de las categorías espaciales, pensar la esencia del tiempo como Devenir (the essence of time as Becoming). Sin embargo, si lo Absoluto no es un principio de estasis (stasis) espacial, si es una modalidad temporal, la complejidad interna de la temporalidad, entonces la satisfacción ya no es portadora del significado de finalidad, estasis y cierre (closure). (SD, pp. 45-46; traducción modificada) ${ }^{17}$.

La apertura sistemática sugiere la apertura en el plano lógico-semántico. Lo Absoluto de Hegel, considerado desde este punto de vista, es la infinitud temporal de las las relaciones lógicas y semánticas del concepto. La base de esta interpretación es la convicción butleriana sobre la falta de cierre tanto de la experiencia fenomenológica como de su exposición. Al respecto, afirma:

En el caso de la Fenomenología, se alcanza lo Absoluto cuando se reconstituye la historia (history) del sujeto viajero como inmanente al sujeto mismo, pero el momento de esa reconstitución no está fuera de la historia (history itself), ni es posible reproducir esa historia en un momento único. Así, la constitución subjetiva de lo Absoluto tiene lugar a través de una serie de actos que son, en principio, infinitos; la tarea fenomenológica de reproducir esa historia será necesaria mientras el sujeto exista en el tiempo, es decir, mientras sea preciso integrar nuevos momentos de la experiencia a la identidad de ese sujeto. Es más, la experiencia nueva no provocará la ampliación de un sujeto existente, sino que origina un relato totalmente nuevo (new narrative) del propio sujeto, un nuevo punto de vista desde el cual es necesario volver a narrar el relato. (SD, pp. 40-41)

Desde este punto de vista interpretativo, la totalidad de las determinaciones conceptuales debería comprenderse como temporalmente infinita y sujeta a las modificaciones históricas acaecidas en la experiencia de la conciencia. Por esto, la proposición "la sustancia es sujeto" y los términos que la componen significan de manera indefinida, así como también el resto de las proposiciones filosóficas que adquieren su sentido especulativo en relación con la totalidad de las relaciones conceptuales. En este sentido se comprende que, para la lectura de Butler, el sujeto gramatical y el humano se hallen en un constante y permanente desplazamiento sin restauración definitiva, esto es, abiertos a la infinitud del movimiento conceptual.

\section{CONCLUSIÓN}

Lo primero que nos gustaría señalar respecto de la interpretación retórica que Butler realiza de la "proposición especulativa" es que el término "Rhetorik" aparece sólo una vez en la $P h$. y con un sentido muy diferente ella le otorga. Hegel lo usa en la "Vorrede" con una acepción negativa y peyorativa para criticar el estado actual del "filosofar natural", que sólo expresa verdaderas triviales e insignificantes $(P h ., 47)$. Por esta razón, comprender la proposición especulativa desde un lineamiento retórico es introducir una clave hermenéutica ajena al propio texto, incorporando aspectos y elementos externos al idealismo especulativo. Sin embargo, y como esperamos haber puesto de relieve en 2.1, aún desde este posicionamiento contemporáneo y extraño a la 
propia terminología hegeliana, la lectura de Butler acuerda con los puntos centrales de la doctrina de la proposición especulativa señalados por parte de la Hegelsforschung $[I]$.

El mayor problema de la propuesta interpretativa de Butler reside, a nuestro entender, en la idea de la apertura lógico-semántica del concepto, que ella fundamenta a partir de la noción temporal de lo Absoluto y desde la falta de cierre de la experiencia fenomenológica y de su exposición narrativa [2. 2].

El tema de la temporalidad en Hegel es sumamente amplio y su tratamiento en la $P h$. no está exento de la complejidad que acompaña a esta problemática. En la obra de 1807 pueden distinguirse diversas concepciones del "tiempo histórico". En la "Vorrede" a la Ph., la "historia universal" (Weltgeschichte) constituye el desarrollo de la cultura de formación (Bildung) del individuo hacia la Ciencia. En esta misma dirección, en la "Einleitung" se sostiene que la sucesión de las figuras del saber conforman la historia detallada (ausführliche Geschichte) de la formación de la conciencia natural hacia el Saber absoluto $(P h ., 56)$. En palabras del filósofo:

La ciencia expone tanto este movimiento formativo en su detalle y su necesidad como expone en su configuración aquello que ya ha descendido a ser momento y patrimonio del espíritu. La meta es que el espíritu llegue a la intelección de lo que es el saber [...] Dado que la substancia del individuo, dado que el espíritu del mundo ha tenido la paciencia de atravesar estas formas [sc. los momentos de su formación] en toda la larga extensión del tiempo (in der langen Ausdehnung der Zeit) y de tomar sobre sí el enorme trabajo de la historia universal (Weltgeschichte), y dado que con menos trabajo que ese el espíritu no puede haber alcanzado ninguna conciencia sobre sí, tampoco el individuo, ciertamente, puede concebir con menos que eso su substancia. Pero, a la vez, entretanto, le cuesta menos esfuerzo porque, en sí, esto ya se ha llevado a cabo [...] La serie de sus configuraciones, que la conciencia va recorriendo por este camino es historia exhaustiva (ausführliche Geschichte) de la cultura como formación (Bildung) de la conciencia misma, hasta llegar a la ciencia. (Ph., 25-26, 56; el subrayado es del original)

La historia (Geschichte) expuesta en la $P h$. no equivale a las verdades historiográficas (historische Wahrheiten). Estas últimas, según Hegel, "conciernen a la existencia singular, a un contenido bajo el aspecto de su contingencia y arbitrariedad, a determinaciones suyas que no son necesarias" (Ph., 31). A diferencia de la Historie, "narración y conocimiento de lo acontecido", la Geschichte es la historia acontecida propiamente dicha, es decir, el verdadero saber histórico que contempla el carácter necesario de la historia de la formación del espíritu (Gómez Ramos 2010, p. 99, n. 49). La historia (Geschichte), en este sentido, es la historia del Espíritu. Es éste el que se despliega históricamente, el que se exterioriza y objetiva en el tiempo, y de ese modo alcanza el saber de sí mismo. Al respecto, Hegel sostiene:

El tiempo (Zeit) es el concepto mismo que está ahí (der daseinde Begrifft selbst) [...] Por lo que concierne, empero, a la existencia ahí (Dasein) de este concepto, la ciencia no aparece en el tiempo y en la realidad efectiva antes de que el espíritu haya llegado hasta esta conciencia acerca de sí. En cuando espíritu que sabe lo que es, no existe antes, y, por lo demás, tampoco en ningún sitio más que después de haber acabado y consumado su trabajo, haber doblegado lo imperfecto de su configuración, haberse procurado para su conciencia la figura de su esencia, y, de esa guisa, haber igualado su autoconciencia con su conciencia [...] El movimiento de hacer brotar a partir de sí la forma de su saber es el trabajo que el espíritu lleva a cabo como historia realmente efectiva (als wirkliche Geschichte) [...] La historia (Geschichte) es el devenir que sabe y se media: el espíritu despojado y exteriorizado en el tiempo; más este exteriorizarse es también el despojamiento de 
sí mismo; lo negativo es lo negativo de sí mismo. Este devenir expone un parsimonioso movimiento y sucesión de espíritus, una galería de imágenes, cada una de las cuales se halla dotada de toda la riqueza íntegra del espíritu, y se mueve con tanta parsimonia precisamente porque el sí-mismo ha de penetrar y digerir toda esta riqueza de su sustancia. En tanto que su compleción consiste en saber perfectamente lo que él es, su substancia, este saber es su ir-dentro-de-sí en el que abandona su existencia y entrega su figura al recuerdo y la interiorización. En su ir-dentro-de-sí, se ha sumergido en la noche de su autoconciencia, pero su desaparecida existencia está preservada dentro de esa noche, y esta existencia cancelada y asumida -que es la anterior, pero renacida a partir del saber- es la nueva existencia, un nuevo mundo y una nueva figura del espíritu. ( $P h ., 34,428$, 430, 433; el subrayado es del original)

El tiempo en tanto que ser-ahí (Dasein) del concepto es la objetivación del espíritu, su exteriorización o existencia temporal. A partir de ésta y mediante la serie de sus configuraciones, el espíritu, como hemos dicho, toma conciencia de sí y alcanza el saber de lo que él es. Ahora bien, esta exteriorización espiritual en el tiempo no es lo que, para Hegel, constituye la "verdadera historia". Siguiendo a De la Maza (2007), se pueden distinguir en la $P h$. tres formas particulares del "tiempo histórico del espíritu". La primera es la de las verdades historiográficas a las que ya nos referimos: la cronología de hechos históricos finitos y contingentes. La segunda es la "historia fenomenológica, que digiere y organiza conceptualmente aquella historia contingente y realiza la mediación con la eternidad [...], tiempo interiorizado o reflexionado en sí por medio del pensamiento" (2007, pp. 7, 9). Esta historia es la historia realmente efectiva que la $P h$. desarrolla conceptualmente mediante la exposición de la experiencia de la conciencia hacia el saber absoluto; es el destino del espíritu que aún no ha alcanzado el saber verdadero de lo que él es. La tercera es "la historia concebida o eternidad mediada con el tiempo finito" (2007, p. 7). La historia concebida (begriffne Geschichte) es la historia en sentido estricto. En otros términos, la noción más elevada del "tiempo histórico" que aparece en el texto de 1807. Sobre el vínculo entre las dos últimas nociones de tiempo histórico, De la Maza sostiene:

Sometido al pensamiento conceptualizador, el pasado histórico efectivo se interioriza, deja de ser mero objeto de intuición, y reúne los momentos esenciales de la experiencia histórica en una totalidad con sentido que sigue siendo temporal, pero una forma de temporalidad cualitativamente nueva, pues se encuentra ahora mediatizada con la eternidad, y en esa medida superada, pero no eliminada. Es una temporalidad cuyo curso queda determinado por el automovimiento del espíritu mismo; ya no es espíritu en el tiempo, sino espíritu como tiempo. El paso de la primera forma a la segunda es llamado por Hegel un "intuir concebido y concipiente". La organización conceptual operada por la Fenomenología crea, entonces, las condiciones que hacen posible una tercera forma de historia del espíritu que sólo es anunciada en la Fenomenología, pero que no corresponde desarrollarla en ella, sino en el sistema de la filosofía al que allana el camino. Se trata de la "historia concebida", en la cual el tiempo se "acaba". (2007, p. 9)

La idea del "acabamiento" del tiempo ha generado no sólo grandes discusiones en la Hegelsforschung, sino también en la filosofía contemporánea que, de una u otra manera, mantiene diversos vínculos con el pensamiento de Hegel. La cuestión reside en cómo comprender la superación o cancelación del tiempo. A propósito de esto, Hegel sostiene:

Dentro del concepto que se sabe como concepto, por tanto, los momentos entran en escena antes que el todo cumplido y lleno, cuyo llegar a ser es el movimiento de esos momentos. Dentro de la conciencia, en cambio, el todo es antes que los 
momentos, pero sin concebir (unbegriffene). El tiempo es el concepto mismo que existe ahí y que, a los ojos de la conciencia, se representa como intuición vacía; por eso, el espíritu aparece necesariamente en el tiempo, y aparece en el tiempo mientras no atrape (erfaßt) su concepto puro, esto es, mientras no borre el tiempo (nicht die Zeit tilgt). Éste es el sí-mismo puro externo, intuido, y no atrapado por el sí-mismo, es el concepto solamente intuido; éste último, en tanto que se atrapa a sí mismo, cancela y asume su forma temporal. Concibe el intuir y es intuir concebido y concipiente (begreift das Anschauen und ist begriffenes und begreifenden Anschauen). De ahí que el tiempo aparezca como el destino y la necesidad del espíritu que no está acabado y completo dentro de sí: la necesidad de enriquecer la parte que la autoconciencia tiene en la conciencia, de poner en movimiento la inmediatez de lo en-sí, la forma en la que la substancia está dentro de la conciencia, o a la inversa, si se toma lo en -sí como lo interior, la necesidad de realizar y revelar lo que primero es sólo interior: esto es, de reivindicárselo a la certeza de sí mismo [...] La meta, el saber absoluto, o el espíritu que se sabe como espíritu, tiene como su camino el recuerdo, la interiorización de los espíritus tal como son en ellos mismos y llevan a cabo la organización de su reino. Su preservación, por el lado de su existencia libre que aparece en la forma de contingencia, es la historia (Geschichte), mientras que por el lado de su organización concebida, es la ciencia del saber que aparece (Wissenschaft des erscheinenden Wissens); tomadas ambas conjuntamente, son la historia comprendida conceptualmente (die begriffene Geschichte), y forman el recuerdo y el calvario del espíritu absoluto, la realidad efectiva, la verdad y la certeza de su trono; sin el cual él sería lo solitario sin vida; y sólo del cáliz de este reino de los espíritu le rebosa la espuma de su infinitud (Unendlichkeit). (Ph., 429, 433-434; el subrayado es del original)

Como bien ha señalado Brauer (1982, pp. 171-195), con quien coincide Bouton en un texto más reciente (2000, pp. 239-294), la supuesta "eliminación" del tiempo que tendría lugar con la llega al Saber absoluto no implica la cancelación de la temporalidad. Aquella debe comprenderse más bien como el pasaje del orden cronológico de la temporalidad al orden lógico del concepto ${ }^{18}$. La historia concebida no es el acabamiento del tiempo, ni el fin de la historia, sino el cumplimiento del tiempo en cuanto el espíritu ha alcanzado el verdadero saber de sí mismo. En este sentido, la historia concebida es la inauguración de un nuevo tiempo histórico que conlleva otra manera de pensar el tiempo. En efecto, en esta nueva época el espíritu ha alcanzado la translucidez conceptual de lo que él mismo es. A propósito de esta cuestión, Hegel sostiene:

No es difícil ver, por lo demás, que nuestro tiempo (unsere Zeit) es un tiempo de parto y de transición (Übergangs) hacia un período nuevo (neuen Periode). El espíritu ha roto con el mundo anterior de su existencia y de sus representaciones, y está a punto de arrojarlo para que se hunda en el pasado, está en el trabajo de reconfigurarse (Arbeit seiner Umgestaltung). Cierto es que él nunca está en calma (Ruhe), sino que está prendido en un permanente movimiento hacia adelante (in immer fortschreiben Bewegung begriffen) [...] El espíritu que se está formando madura lenta y silenciosamente hacia la nueva figura, disuelve trozo a trozo la arquitectura de su mundo precedente, cuyo tambalearse viene indicado sólo por unos pocos síntomas sueltos; la frivolidad y el tedio que irrumpen en lo existente, el barrunto indeterminado de algo desconocido, son los emisarios de que algo otro está en marcha. Este paulatino desmoronarse que no cambiaba la fisionomía del todo se ve interrumpido por el amanecer, un rayo que planta de golpe la conformación del nuevo mundo (neuen Welt). (Ph., 14-15; el subrayado es del original). 
Esta nuevo período no es ni más ni menos que el del espíritu que, habiendo llegado al saber de sí, se piensa a sí mismo como tal. No se trata ya del espíritu que recorre el camino de su formación. Por esta razón, lo que hace la $P h$. es exponer el recorrido mediante el cual el espíritu alcanza la autoconciencia de sí (el Saber absoluto). Pero no expone en sentido estricto la historia concebida: la filosofía especulativa es la explicitación conceptual del saber que el espíritu tiene de sí.

Ahora bien, la noción de "historia concebida" puede, a la vez, entenderse de diferentes maneras en lo que respecta al problema del cerramiento o la apertura del Sistema. Una primera interpretación consiste en que el sistema de la filosofía se cerró definitivamente con el saber de sí ya alcanzado por el espíritu. Desde esta perspectiva, ningún suceso histórico puede modificar la verdad del sistema expuesta por la filosofía hegeliana. La segunda lectura comprende que la liberación de la contingencia temporal lograda por el Saber absoluto del espíritu permite la apertura de nuevos desarrollos históricos. Desde este punto de vista, los nuevos sucesos históricos pueden comenzar un nuevo período del espíritu y éste deberá volver a liberarse a sí mismo para alcanzar una nueva figura de su verdad, en la que se contemplarían nuevos conceptos filosóficoespirituales. Esta segunda interpretación no niega la sistematicidad de la verdad filosófica, pero tampoco clausura la posibilidad de un recomienzo histórico del espíritu (De la Maza 2007, pp. 19-20).

Nuestro propósito no es tomar posición sobre una de las dos lecturas recién descriptas. Tan sólo queremos señalar que la interpretación de Judith Butler se ubica en la segunda de las dos posiciones antedichas. La idea butleriana sobre la apertura de la metafísica hegeliana es compatible con ciertas lecturas actuales, según las cuales, el Saber absoluto, en palabras de Jarczyk y Labarrière, "no es lo absoluto del saber" (n'est pas l'absolu du savoir) (1996, pp. 9-14, 217-230) ${ }^{19}$. Sin embargo, a la luz de los contenidos expuestos es menester enfatizar que la propuesta hermenéutica de la filósofa norteamericana parece limitarse al tiempo histórico fenomenológico, sin dejar en claro su paso a la historia concebida y su relación. Entendemos que esta restricción se explica por el hecho de que la hermenéutica general que Butler realiza de la $P h$. en $S D$ se limita fundamentalmente a la figura de la Autonconciencia, sin tomar en consideración las mediaciones subsiguientes. Esto conlleva que sus tesis sobre la apertura del sistema hegeliano se vean limitadas por su propio recorte interpretativo, aunque no por esto, entendemos, sus ideas carecen de todo tipo de valor hermenéutico.

\section{REFERÉNCIAS}

ABELLÓN, Pamela, CHIACCHIO, Cecilia, FEMENÍAS, María Luisa. "Breve recorrido sobre la influencia de Hegel en la filosofía de Judith Butler. Entrevista a Judith Butler". Avatares filosóficos, N. ${ }^{\circ} 3$., pp. 185-192, 2016.

ABELLÓN, Pamela. "Judith Butler hace de Georg Hegel un filósofo intempestivo. Performatividad y pérdida de sí en la Phänomenologie des Geistes". En FEMENÍAS, María Luisa, MARTÍNEZ, Ariel (Comps.); Judith Butler: la opacidad del sujeto. La Plata: Edulp, 2015. pp. 41-72.

BOUTON, Christophe; Temps et esprit dans la philosophie de Hegel. De Frankfort à Iéna. París: Vrin, 2000.

BRAUER, Oscar Daniel; Dialektik der Zeit, Untersuchungen zu Hegels Metaphysik der Zeit. Stuttgart: Frommann-Holzboog, 1982.

BURGOS, Elvira; Qué cuenta como una vida. La pregunta por la libertad en Judith Butler. Madrid: Mínimo tránsito, 2008.

BUTLER, Judith; Subjects of Desire. Hegelian Reflections in Twentieth-Century France. New York: Columbia University Press, 1987 (1999) (Sujetos del Deseo. 
Reflexiones hegelianas en la Francia del siglo XX. Trad. de E. L. Odriozola. Buenos Aires: Amorrortu, 2012.

"Geist ist Zeit: French Interpretations of Hegel's Absolute". Berkshire Review, $\mathrm{N}^{\circ}$ 20, pp. 66-80, 1985.

CUBO UGARTE, Oscar; Actualidad hermenéutica del "Saber Absoluto". Una lectura de la Fenomenología del espíritu de Hegel. Madrid: Dykinson, 2010.

DE LA MAZA, Luis Mariano. "Tiempo e historia en la Fenomenología del espíritu de Hegel”. Ideas y Valores, $\mathrm{N}^{\circ}$ 133, pp. 3-22, 2007.

DUQUE, Félix. "Estudio preliminar". En HEGEL, Georg Wilhelm Friedrich; Ciencia de la lógica (I). Trad. F. Duque. Madrid: Abada editores y UAM ediciones, 2011. pp. 13-182.

Hegel. La especulación de la indigencia. Barcelona: Granica, 1990.

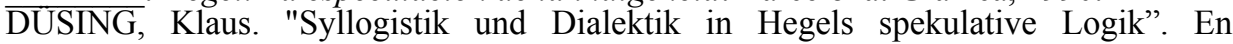
HENRICH, Dieter (ed.). Hegels Wissenschaft der Logik: Formation und Rekonstruktion. Stuttgart: Klett-Cotta, 1986. pp. 15-38.

GÓMEZ RAMOS, A. "Presentación". En HEGEL, Georg Wilhelm Friedrich. Fenomenología del espíritu. Trad. de A. Gómez Ramos (Ed. bilingüe), Madrid: Abada, 2010. pp. 7-48.

HEGEL, Georg Wilhelm Friedrich. "Differenz des Fichte'shen und Schelling'schen Systems der Philosophie". En HEGEL, Georg Wilhelm Friedrich. Jenaer kritische Schriften (1801-1807). Werke in 20 Bänden. Frankfurt am Main: Suhrkamp, 1970, Vol. 2 (Diferencia entre el sistema de filosofía de Fichte y el de Schelling. Trad. de A. Rodríguez Tous. Madrid: Alianza, 1989).

Phänomenologie des Geistes. Publicada por primera vez bajo el título System der Wissenschaft. Erster Theil, Die Phänomenologie des Geistes. Bamberg und Würzburg, 1807 (Fenomenología del espíritu. Edición bilingüe. Trad. de A. Gómez Ramos. Madrid: Abada, 2010; Phenomenology of Spirit. Trad. de A. V. Miller. Oxford: Clarendon Press, 1977).

Wissenschaft der Logik I. En Werke in 20 Bänden. Frankfurt am Main: Suhrkamp, 1970, Vol. 5 (Ciencia de la lógica I. Trad. F. Duque. Madrid: Abada editores y UAM ediciones, 2011).

Enzyklopädie der philosophischen Wissenschaften im Grundrisse. En Werke in 20 Bänden. Frankfurt am Main: Suhrkamp, 1970, Vols. 8-10 (Enciclopedia de las ciencias filosóficas en compendio. Trad. de R. Valls Plana. Madrid: Alianza, 2005).

. Briefe von und an Hegel. Hamburgo: Ed. Hofmeister, 1952, Vol. 1.

HOFFMANN, Thomas Sören; Georg Wilhelm Friedrich Hegel: Eine Propädeutik. Wiesbaden: Marixverlag, 2004 (Hegel. Una propedéutica. Trad. de M. Mueriera y K. Wrehde. Buenos Aires: Biblios, 2014).

JARCZYK, Gwendoline y LABARRIÈRE, Pierre Jean; De Kojève à Hegel. Cent cinquante ans de pensée hégélienne en France. Paris: Albin Michel, 1996.

LABARRIERE, Pierre Jean. "La sursomption du temps et le vrai sens de l'histoire conçue". Revue de Métaphysique et de Morale, 84e Année, $\mathrm{N}^{\circ}$ 1, pp. 92-100, 1979.

LAU, Chong-Fuk. "Language and Metaphysics: The Dialectics of Hegel's Speculative Proposition". En O’NEILL SURBER, Jere (Ed.). Hegel and Language. New York: State University of New York Press, 2006. pp. 55-74.

LÓPEZ, Diana. "La construcción lógica de la Fenomenología del Espíritu de Hegel". Tópicos, N. ${ }^{\circ}$ 30, pp. 40-63, 2015.

MALABOU, Catherine: L'Avenir de Hegel. Plasticité, temporalité, dialectique. París: Vrin, 1996 (The Future of Hegel. Plasticity, Temporality and Dialectic. Trad. de L. During. London-New York: Routledge, 2005).

MCCUMBER, John; The Company Words. Hegel, Language, and Systematic Philosophy. Evanston - Illinois: Northwestern University Press, 1993.

MONTECINOS, Sergio. "Autoconsumación del escepticismo y proposición 
especulativa. El alcance metodológico de dos conceptos claves de la Fenomenología del espíritu". Bajo palabra. Revista de Filosofía, Vol. 2, $\mathrm{N}^{\circ} 7$, pp. 51-62, 2012.

NICOLIN, Friedhelm, "Das Titelproblem der Phänomenologie des Geistes". HegelStudien, Vol. 4, pp. 113-123, 1967.

NUZZO, Angelica. “The Language of Hegel's Speculative Philosophy”. En O'NEILL SURBER, Jere (Ed.). Hegel and Language. New York: State University of New York Press, 2006. pp. 75-94.

OLSON, Gary. y WORSHAN, Lynn. "Changing the Subject: Judith Butler's Politics of Radical Resignification". Jac, Vol. 20, N 4, pp. 727-765, 2000 ("Cambio de sujeto: la política de la resignificación radical de Judith Butler”. En CASALE, Rolando, CHIACCHIO, Cecilia, Máscaras del deseo. Una lectura del deseo en Judith Butler. Buenos Aires: Catálogos, 2009, pp. 65-112).

ROSENKRANZ, Karl; Georg Wilhelm Friedrich Hegel's Leben. Berlin: Duncker und Humblot, 1844.

RÜHLE, Volker; "El prólogo de Hegel a la Fenomenología del espíritu”. En DUQUE, Felix. (Ed.). Hegel. La odisea del espíritu. Madrid: Ediciones Pensamiento, 2010. pp. 15-34.

SALIH, Sara. Judith Butler. London-New York: Routledge, 2002.

SÁNCHEZ FERNÁNDEZ, José Manuel, "El problema de la mediación desde la perspectiva de la ciencia de la experiencia de la conciencia". Arhe, Vol. III, N ${ }^{\circ} 5$ 6, pp. 125-146, 2006.

SIEP, Ludwig, Der Weg der Phänomenologie des Geistes. Ein einführender Kommentar zu Hegels 'Differenzschrift' und 'Phänomenologie des Geistes'. Frankfurt am Main: Suhrkamp, 2000 (El camino de la Fenomenología del espíritu,. Un comentario introductorio al Escrito sobre la diferencia y la Fenomenología del espíritu de Hegel. Trad. de Carlos Rendón. México: Antphropos, 2015).

SIMON, Josef; Das Problem der Sprache bei Hegel. Stuttgart-Berlin: Kohlhammer, 1966 (El problema del lenguaje en Hegel. Trad. Ana Agud. Madrid: Taurus, 1982).

SURBER, Jere Paul. "Hegel's Speculative Sentence”. Hegel-Studien, Vol. 10, pp. 2130, 1975.

VICKI, Kirby; Judith Butler: Live Theory. London-New York: Continuum, 2006.

WOHLFART, Gunter; Der spekulative Satz. Berlin: Gruyter, 1981.

\section{Notas}

1 Las referencias de la $P h$. corresponden a la numeración canónica de la traducción castellana de Gómez Ramos (Abada, 2010), que sigue la edición canónica de Bonsiepen y Heede (Gesammelte Werke, Band 9, Hamburg, 1980), aunque también toma en consideración la versión de 1988 de Felix Meiner Verlag y la versión digital de la edición de Schulze de 1832, a la que suma algunas modificaciones.

2 Independientemente del término "spekulativer Satz", el concepto de la proposición especulativa se encontraría también en escritos anteriores a la $P h$., en especial en los apartados "Verhältnis der Spekulation zum gesunden Menschenverstand" y "Prinzip einer Philosophie in der Form eines absoluten Grundsatzes" de "Differenz des Fichte'shen und Schelling'schen Systems der Philosophie” (pp. 21-28; Sánchez Fernández 2009, pp. 8788).

3 El libro completo se tituló System der Wissenschaft. Erster Theil, Die Phänomenologie des Geistes. Cabe recordar, además, que la "Vorrede" fue escrita entre diciembre y enero de 1807, es decir, unos meses después de terminado el libro en cuestión. Existe una idea bastante difundida, basada en una carta que Hegel le escribe a Schelling en mayo de 1807, según la cual, la $P h$. queda terminada en la medianoche del 13 de octubre de 1806, el día anterior al comienzo de la batalla de Jena (Briefe von und an Hegel, Ed. 
Hofmeister, Hamburgo, 1952, Vol. 1, pp. 161 y ss.). Gómez Ramos pone en cuestión tal opinión y sostiene que para esa fecha el texto ya había sido escrito (2010, pp. 17-18). Según esta segunda versión, Hegel huye de su casa el día 13 de octubre debido al saqueo de los soldados franceses y se hospeda en la del comisario Hellfeld, en Bamberg. Desde allí le escribe a su amigo Niethammer, comentándole que ya había enviado al editor una parte del manuscrito, aunque temía que no pudiera publicarse debido a las condiciones bélicas de entonces (Rosenkranz 1844, p. 229). Para el complejo proceso de redacción de la $P h$. y el problema del título, vésase Nicolin (1967) y López (2015).

4 Esta segunda opinión toma como base algunas afirmaciones sobre la incapacidad de la forma proposicional para expresar las verdades especulativas que Hegel formula en la $E P W(\S \S$ $31,88)$ y en la Wissenschaft der Logik I $(49,54)$.

$5 \mathrm{El}$ tratamiento de Butler sobre la proposición especulativa se encuentra específicamente en los dos Prefacios $(1987,1999)$, la Introducción y el Capítulo I de $S D$.

6 Escribimos "presentación" entre comillas porque para Hegel, como es sabido, la filosofía especulativa no puede presentarse en el sentido tradicional y ordinario del término, sino tan sólo exponerse.

7 La expresión de Hegel es: "Daß dem nicht so sei, diese Einsicht ist das bloß Negative" (Ph., LXXIII; el subrayado es del original). La traducción de Gómez Ramos es la siguiente: "Decir que algo no es así: esa intelección es lo meramente negativo" (Ph., 42; el subrayado es del original).

8 Esto retoma la idea según la cual: "La verdadera figura en la que existe la verdad sólo puede ser el sistema filosófico de la misma [...] y la única explicación (Erklärung) satisfactoria a este respecto es la exposición de la filosofía misma (die Darstellung der Philosophie selbst)" (Ph., 11).

9 La distinción entre lo mentado o supuesto y lo realmente dicho es también señalada por Félix Duque (1990). Según este autor, la proposición especulativa no puede expresar -en el sentido de "decir"- las determinaciones simples del concepto, sino sólo indicarlas (1990, p. 31).

10 "Exposition" es el término con el que, en la edición inglesa de la $P h$., Miller traduce el término alemán "Darstellung" (1977, $\S \S 5,76-77)$. En la versión castellana de $S D$, Odriozola traduce el pronombre posesivo "its" por el pronombre demostrativo "esa", mientras nosotros lo hacemos por "su".

11 Los rasgos activos del lenguaje hegeliano que Butler señala en afirmaciones como ésta constituyen para Burgos (2008, pp. 14-16) y Salih (2002, pp. 13-15) elementos comunes con la propia teoría de la performatividad linguística que Butler defiende en obras posteriores a $S D$. Nosotros abordamos de modo incipiente del aspecto performativo que para Butler tendría el lenguaje de Hegel en Abellón (2015). En la presente investigación no retomamos esta cuestión puesto que para ello deberíamos realizar una serie de precisiones y profundizaciones que nos alejarían de nuestros presentes objetivos. En una entrevista a Butler que hemos tenido oportunidad de realizar junto con Femenías y Chiacchio, ella sostiene al respecto: “- Entrevistadoras: ¿Qué relación se puede establecer entre su abordaje retórico de la proposición especulativa de Hegel y su propia noción de la performatividad lingüística? - J. B.: Por supuesto, la idea de actos de habla performativos viene de una tradición diferente, la de J. L. Austin, y por ello en un principio parece difícil trazar analogías entre Austin y Hegel. Pero si consultamos la lectura que Derrida hace de Austin, o la que hace Shoshana Felman de Austin, podemos ver que el acto de habla actúa de una forma que no siempre es anticipada. Derrida llama nuestra atención sobre el carácter iterable del acto de habla y sugiere que su instancia puntual puede ser entendida sólo como una repetición de instancias anteriores. Aunque su explicación de la iterabilidad es formal y concierne a la forma en que el significante llega a actuar a través del tiempo, me hago una pregunta diferente: qué clase de patrones sociales o rituales se presuponen y se reinvocan en el acto de habla. Con una formulación diferente, Bourdieu hace la misma pregunta, pero para él sólo las convenciones existentes condicionan los actos de habla; en cambio, a mí me interesa el carácter no concluyente de la iterabilidad así como la posibilidad de generar nuevas convenciones sobre la base de 
las prácticas iterables. Por su lado, la lectura de Felman nos permite ver que el cuerpo siempre está implicado en el acto de habla, como lo están la vida del deseo y el inconsciente; la idea de ritual también nos permite comprender los actos corporales como performativos. Por lo tanto, el entrecruzamiento entre lo corporal y lo lingüístico es crucial para mi concepto de performatividad. Hegel es interesante aquí, pues en la Fenomenología puntualiza constantemente, en especial en la sección de la percepción sensible, cómo varias declaraciones sobre lo que constituye la realidad última se rechazan a sí mismas. Si decimos que la inmediatez es verdad y que la condición de la afirmación es la mediación, entonces podemos hacer la afirmación, pero esto involucra al hablante en una contradicción performativa. Ciertamente, la lectura de la Fenomenología es un proceso de experimentar una serie de falsas afirmaciones sólo para ganar un sentido de verdad más expansivo. De esta manera, el resultado inesperado del acto de habla nos vuelve a traer al Austin de Derrida. Y el hecho de que debamos experimentar o someternos al acto de habla implica una dimensión corporal del acto de habla" (Abellón, Chiacchio y Femenías 2016, p. 188).

12 La negación, en efecto, también está presente en el sentido indicado en aquellos casos en los que el verbo principal de la oración es el verbo "ser". A propósito de esto, Butler sostiene: "Cuando 'ser' es el verbo principal en alguna afirmación, muy pocas veces es portador de la carga conocida de la predicación; más bien se vuelve transitivo, de una manera desconocida y amenazante, y afirma el movimiento inherente del ser, al tiempo que trastoca los supuestos ontológicos que el uso habitual del lenguaje nos induce a adoptar" (SD, p. 50).

13 Butler reitera esta idea en la entrevista que le realizan Olson y Worsham en el año 2000. Una de las primeras preguntas que le dirigen hace referencia a su forma peculiar de escritura, la cual ha sido ferozmente criticada por Martha Nussbaum en un artículo publicado en New Republic el año anterior y que se titutó 'The Professor of Parody'. En este marco Butler vuelve sobre el tema de la proposición especulativa de Hegel, afirmando: "Me formé en la filosofía continental y eso significó pasar buena parte del tiempo leyendo a Hegel y asistir a numerosos seminarios sobre Heidegger. En las concepciones filosóficas que se expresaban en esas lecciones, la dificultad del lenguaje era en cierta forma esencial. Por ejemplo, cuando Hegel habla sobre la 'oración especulativa', está obrando contra la forma proposicional tal cual se recibe. Cuando dice 'El sujeto es el espíritu', la primera inclinación, aquella para la que la gramática aceptada (received grammar) nos prepara, es establecer 'el sujeto' como sujeto de la oración, y luego 'el espíritu' se vuelve una forma de calificar o determinar a aquel sujeto. Pero, por supuesto, busca que seamos capaces de revertir esa oración, reconocer algo acerca de cómo funciona el 'es': que no apunta literalmente en una dirección; por el contrario, apunta en ambas direcciones a la vez. Quiere que seamos capaces de experimentar la simultaneidad de esa oración, su funcionamiento en una doble direccionalidad. Ahora bien, eso es muy difícil de hacer, pues debemos ver cuánto nos inclinamos a lo que Nietzsche llamó 'la seducción de la gramática', es decir, a leer en una forma lineal [...] Entonces, parte de mi propia formación era entregarme a los desafíos gramaticales profundos -desafíos de gramática, desafíos del lenguaje ordinario- y esto era muy estimulante. Incluso, diría que esos textos representaban en cierta forma el modernismo tardío de la tradición filosófica continental [...] Hay algo en la vida de la oración que se vuelve nuevo, o raro, o extraño en una forma fundamental y eso es lo que yo fui a buscar. Me seducía una noción que considero modernista; la idea de que, entrometerse con la gramática tal cual se la había recibido, iba a descubrir algo nuevo del mundo" (Olson, G. y Worshan [2000] 2009, pp. 68-69).

14 Cabe resaltar que, en este sentido, el holismo no se restringe a una cuestión meramente lingüística y semántica sino que, al ser la expresión de las determinaciones del concepto, compete también a la ontología. A propósito de esta cuestión, Siep sostiene: "Holismo y forma de sistema se enlazan no sólo en orden a una peculiaridad de nuestro saber, a una forma de organización de teorías o principios verdaderos sobre la realidad, sino que esta forma le corresponde a la realidad. 'Sistema', 'sujeto', 'espíritu' expresan, en la citada fórmula, la substancia o lo absoluto, es decir, la realidad misma propiamente dicha 
([2000] 2015, p. 68). La fórmula a la que Siep se refiere es la siguiente: "Que lo verdadero sólo en cuanto sistema es efectivamente real, o bien, que la substancia es esencialmente sujeto (die Substanz wesentlich Subjekt ist), queda expresado en la representación que enuncia lo absoluto como espíritu (das Absolute als Geist)" (Ph., 22; el subrayado es del original).

15 Lo que nosotros traducimos por "sujeto vivo" corresponde a "living subject", término que Oriozola traduce por "ser vivo".

16 Butler desarrolla este tema en "Geist ist Zeit: French Interpretations of Hegel's Absolute". Este texto se publicó en el año 1985 en Berkshire Review, $\mathrm{N}^{\circ}$ 20, pp. 66-80, y nos ha sido imposible acceder a él. En una comunicación directa con Butler, la autora nos confirmó que ella tampoco dispone del artículo. Suponemos que el texto, que nunca fue digitalizado, lamentablemente se perdió. Sin embargo, la tesis central de "Geist ist Zeit" se retoma en $S D$. En el presente trabajo, reponemos sólo la idea general de esta cuestión, puesto que de lo contrario nos alejaríamos de nuestros objetivos específicos.

17 "Openness", que nosotros traducimos por "apertura", Odriozola lo hace por "imparcialidad".

18 Una interpretación que toma otra perspectiva pero que comparte este lineamiento general es la de Labarrière (1979).

19 Una idea similar se encuentra en Simon ([1966] 1982, pp. 255-258). En esta misma línea parece inscribirse la propuesta de Hoffmann (2004). Al respecto, el autor sostiene: "El hegeliano concepto 'temporalizado' de filosofía y verdad parece estar en tensión con el hecho de que su filosofía no sólo no evita el concepto del absoluto, sino que está centrada en él, su concepto es el terrateniente lógicamente inevitable de la totalidad; y en la medida en que la filosofía de Hegel está en deuda con la necesidad de explicar la totalidad, así también es la explicación del absoluto. Visto más de cerca, en la tensión entre temporalidad y absolutez, en cuanto dato clave del pensamiento filosófico, no se trata precisamente de una contradicción insoluble [...] La filosofía no llega a ser concreta 'más acá' de un absoluto intocable e inexplicable en sí, sino que ella misma es el entrar del absoluto, de la totalidad en el tiempo, en su 'encarnación' [...] La filosofía es, por fin, la conciencia determinada de que, en cada momento, es posible el 'tiempo cumplido' en el terreno del saber, como real fue también siempre en la historia de la filosofía. De una manera similar a como el concepto de la totalidad de Hegel no amputó a la omnisciencia, su concepto de lo absoluto, recogido filosóficamente, no está en camino a ninguna perfección técnica del conocer. Él va camino a una autoreferencia estricta de la relación cognitiva, que, en ningún sentido, ya está arrojada fuera de sí y que, de este modo, alcanza una intensidad que ya no es posible para la conciencia sencilla, orientada objetualmente (y por eso relativa) [...] Según Hegel, los puntos centrales de la historia del conocimiento tienen entonces innegablemente su dialéctica, y nadie tiene el permiso para declarar en un sentido abstracto cuál es ‘el último' o el fin de la historia. Pero consta que una participación dialéctica de una 'historia de lo absoluto' ciertamente no es una objeción contra el sentido absoluto de cada participante singular, aunque la existencia de la libertad del otro es, por cierto, una objeción contra la propia. Que 'lo absoluto', en fin, no es sólo un fundamento y un punto de partida, sino indudablemente también meta y medida interior del concepto hegeliano de filosofía, no significa, por su lado, ninguna separación de la filosofía de las constelaciones históricamente concretas, en las cuales ella reside. Tampoco Hegel dispuso, para todos los tiempos, la contextura filosófica para dejar a los posteriores sólo la repetición. Si 'lo absoluto' designa la exigencia de la filosofía de constituir en el camino del pensar el tiempo cumplido [...], entonces radica precisamente en ello un impulso que, ciertamente, no puede ser satisfecho con la constatación de lo que ha sido. Esto por cierto no excluye que, doscientos años después de Hegel, quede todavía muchísimo por aprender si se tiene que tomar en serio este impulso" ([2004] 2014, pp. 45, 47-48; el subrayado es del original). Malabou, desde otro enfoque, centrado fundamentalmente en la $E P W$, entiende el análisis del tiempo y particularmente del porvenir (avenir) en términos de "plasticidad" (plasticité / Plastizität) ([1996] 2005, pp. 1-20, 133-134). 